PROCEEDINGS OF THE

AMERICAN MATHEMATICAL SOCIETY

Volume 133, Number 2, Pages 595-604

S 0002-9939(04)07711-1

Article electronically published on September 20, 2004

\title{
LAGRANGE MULTIPLIERS FOR FUNCTIONS DERIVABLE ALONG DIRECTIONS IN A LINEAR SUBSPACE
}

\author{
LE HAI AN, PHAM XUAN DU, DUONG MINH DUC, AND PHAN VAN TUOC
}

(Communicated by Jonathan M. Borwein)

\begin{abstract}
We prove a Lagrange multipliers theorem for a class of functions that are derivable along directions in a linear subspace of a Banach space where they are defined. Our result is available for topological linear vector spaces and is stronger than the classical one even for two-dimensional spaces, because we only require the differentiablity of functions at critical points. Applying these results we generalize the Lax-Milgram theorem. Some applications in variational inequalities and quasilinear elliptic equations are given.
\end{abstract}

\section{INTRODUCTION}

The concept of Gâteau-differentiability is compatible to weak solutions of partial differential equations but not to generalized solutions of partial differential equations. In some cases the considered functionals are only derivable along directions in a linear subspace of a Banach space where they are defined. In 2, 3, 6, 9, 12, these cases have been studied under some generalized Palais-Smale conditions and mountain-pass lemma techniques. In the present paper we prove a Lagrange multipliers theorem for those functionals. Our result is available for topological linear vector spaces and is stronger than the classical one even for two-dimensional spaces (see [7, p.293), because we only require the differentiablity of functions at critical points.

The essential point of the Lagrange multipliers theorem is to provide the collinearity of two vectors (usually denoted by $D f(x)$ and $D g(x)$ ). We shall reduce this problem to the two-dimensional case by the following idea : Let $x=\left(x_{1}, \cdots, x_{n}\right)$ and $y=\left(y_{1}, \cdots, y_{n}\right)$ be two vectors in $\mathbb{R}^{n}$ with $y_{1} \neq 0$. In order to prove the collinearity of $x$ and $y$ it is sufficient to show that $\left(x_{1}, x_{i}\right)$ and $\left(y_{1}, y_{i}\right)$ are collinear for any $i$ in $\{2, \cdots, n\}$. Indeed $x=\frac{x_{1}}{y_{1}} y$ in this case. Using this reduction we neither need the compactness conditions on functionals nor the completeness of infinite-dimensional spaces.

In the second section we give simple proofs of Lagrange multiplier theorems without any assumption on completeness and local convexity of considered spaces. We only need these results for the Banach spaces in the last two sections, but we do

Received by the editors February 20, 2003.

2000 Mathematics Subject Classification. Primary 58E05, 49J40, 35J25, 35J60.

Key words and phrases. Lagrange multipliers theorem, Lax-Milgram theorem, variational inequalities, quasilinear elliptic eigenvalue problems.

This work was partially supported by CONACyT (Mexico), grant G36357-E. 
not get any extra complication for the proofs in general cases of topological vector spaces.

In the third section we point out that the Lax-Milgram theorem can be obtained by using the Lagrange multiplier theorem, and we generalize its classical version (see 4], p.84), then apply it to solve an elliptic problem.

In the last two sections we apply our results to variational inequalities and quasilinear elliptic eigenvalue problems.

\section{LAGRANGE MULTIPLIERS THEOREMS FOR $G$-DIFFERENTIABLE FUNCTIONS IN TOPOLOGICAL LINEAR SPACES}

Let $U$ be an open subset of a topological vector space $E, G$ be a linear subspace of $E$, and $f$ be a function from $U$ into $\mathbb{R}$. For any $(x, h) \in U \times G$ we define $U(x, h)=\{t \in \mathbb{R}: x+t h \in U\}$ and $f_{(x, h)}(t)=f(x+t h) \forall t \in U(x, h)$. Since $U$ is open in $E$, it is clear that $U(x, h)$ is an open subset of $\mathbb{R}$.

We say : (i) $f$ is $G$-continuous on $U$ if and only if $f_{(x, h)}$ is continuous at 0 for any $(x, h)$ in $U \times G$.

(ii) $f$ is $G$-differentiable at $x$ if and only if there exists a linear mapping $D f(x)$ from $G$ into $\mathbb{R}$ such that

$$
\lim _{t \rightarrow 0} \frac{f(x+t h)-f(x)}{t}=\operatorname{Df}(x)(h) \quad \forall h \in G .
$$

(iii) $f$ is $G$-differentiable on $U$ if $f$ is $G$-differentiable at every $x$ in $U$.

(iv) $f$ is strongly $G$-differentiable at $x$ if and only if there exists a linear mapping $D f(x)$ from $G$ into $\mathbb{R}$ such that

$$
\lim _{(s, t) \rightarrow 0} \frac{f(x+s t h+s k)-f(x)}{s}=\operatorname{Df}(x)(k) \quad \forall h, k \in G .
$$

Example 2.1. Let $G$ be a linear subspace of a topological vector space $E$ and $f$ be a linear mapping from $E$ into $\mathbb{R}$, which may be discontinuous on $E$. It is clear that $f$ is $G$-continuous on $E$. Fix three vectors $x, h$ and $k$ in $E$. We see that

$$
\frac{f(x+s t h+s k)-f(x)}{s}=f(k)+t f(h) \quad \forall s, t \in \mathbb{R} \backslash\{0\} .
$$

Therefore, $f$ is strongly $G$-differentiable at any $x$ in $E$ for any linear subspace $G$ of $E$.

Example 2.2. Let $U$ be an open subset of a normed linear space $E, x$ be in $U$ and $f$ be a mapping from $U$ into $\mathbb{R}$ such that $f$ is Fréchet differentiable at $x$. It is clear that $f$ is strongly $G$-differentiable at $x$ for any linear subspace $G$ of $E$.

First we have an "implicit mapping theorem" for $G$-differentiable mappings.

Lemma 2.3. Let $U$ be an open subset of a topological vector space $X, G$ be a linear subspace of $X, g$ be a real $G$-continuous function on $U$. Let $u$ be in $U$, and $v$ and $w$ be in $G$. Assume that $g$ is $G$-differentiable at $u$ and $D g(u)(w) \neq 0$. Put $\beta=-\frac{D g(u)(v)}{D g(u)(w)}$. Then there exist two sequences of real numbers $\left\{\alpha_{n}\right\}$ and $\left\{\delta_{n}\right\}$ converging to 0 such that $\alpha_{n}$ is positive and $g\left(u_{n}\right)=g(u)$ for any integer $n$, where $u_{n}=u+\alpha_{n}\left(\delta_{n}+\beta\right) w+\alpha_{n} v$. 
Proof. Replacing $g$ with $g-g(u)$ if necessary, we can assume without loss of generality that $g(u)=0$. By definition we see that $D g(u)(\beta w+v)=0$. For sufficiently small real numbers $\alpha$ and $\delta$ we define

$$
\begin{aligned}
\phi(\alpha, \beta, \delta) & =\alpha^{-1}[g(u+\alpha(\delta w+\beta w+v))-g(u)]-D g(u)(\delta w+\beta w+v), \\
G_{\alpha}(\delta) & =g(u+\alpha(\delta w+\beta w+v)) .
\end{aligned}
$$

Since $g(u)=0$ and $g$ is $G$-differentiable at $u$, we have $\lim _{\alpha \rightarrow 0} \phi(\alpha, \beta, \delta)=0$ and

$$
\begin{aligned}
& G_{\alpha}(\delta)=g(u+\alpha(\delta w+\beta w+v))-g(u) \\
& =\alpha[D g(u)(\delta w+\beta w+v)+\phi(\alpha, \beta, \delta)]=\alpha[\delta D g(u)(w)+\phi(\alpha, \beta, \delta)] .
\end{aligned}
$$

Hence there is a sequence of positive real numbers $\left\{\alpha_{n}\right\}$ converging to 0 such that

$$
G_{\alpha_{n}}\left(-\frac{1}{n}\right) \cdot G_{\alpha_{n}}\left(\frac{1}{n}\right)<0 .
$$

By (i) there is an $n_{0}$ such that the map

$$
x \longmapsto G_{\alpha_{n}}(x)=g\left(u+\alpha_{n}(\beta w+v)+x \alpha_{n} w\right)
$$

is continuous on $\left[-\frac{1}{n}, \frac{1}{n}\right]$ for any integer $n \geq n_{0}$. Thus there exists a $\delta_{n}$ in $\left[-\frac{1}{n}, \frac{1}{n}\right]$ such that $G_{\alpha_{n}}\left(\delta_{n}\right)=g\left(u_{n}\right)=0$, where $u_{n}=u+\alpha_{n}\left(\delta_{n} w+\beta w+v\right)=u+$ $\alpha_{n}\left(\delta_{n}+\beta\right) w+\alpha_{n} v$.

Remark 2.4. If $v$ and $w$ are linearly independent, then $u_{n}$ and $u$ are different for any integer $n$.

Let $C$ be a subset of a topological vector space $X$. Then $C$ is called a cone if $\alpha u$ is in $C$ for any $(\alpha, u)$ in $[0, \infty) \times C$. We have the following Lagrange multiplier results for $G$-differentiable functions.

Theorem 2.5. Let $C$ be a convex cone of a topological vector space $X, U$ be an open subset of $X, G$ be a linear subspace of $X, u$ be in $U \cap C \cap G, r$ be a real number, and $f$ and $g$ be two functions on $U$. Put $M=\{x \in C \cap U: g(x)=r\}$. Assume:

(i) $f(u)$ is the minimum (or maximum respectively) of $f(M)$,

(ii) $f$ is strongly $G$-differentiable at $u$ and $g$ is $G$-differentiable at $u$,

(iii) $g$ is $G$-continuous on $U$ and $D g(u)(u) \neq 0$.

Then there exists a real number $\lambda$ such that $\lambda D g(u)(v-u) \leq D f(u)(v-u)$ (or $\lambda D g(u)(v-u) \geq D f(u)(v-u)$ respectively) for any $v$ in $C \cap G$.

Proof. Applying Lemma 2.3 for $w=u$, we can find two sequences of real numbers $\left\{\alpha_{n}\right\}$ and $\left\{\delta_{n}\right\}$ converging to 0 such that $\alpha_{n}$ is positive and $g\left(u_{n}\right)=r$ for any integer $n$, where $u_{n}=\left[1+\alpha_{n}\left(\delta_{n}+\beta\right)\right] u+\alpha_{n} v$ and $\beta=-\frac{D g(u)(v)}{D g(u)(u)}$. Since $C$ is a convex cone and $\left[1+\alpha_{n}\left(\delta_{n}+\beta\right)\right]>0$ for every sufficiently large $n$, we can suppose that $u_{n}$ is in $M$ for any integer $n$, and we have

$$
0 \leq \alpha_{n}^{-1}\left[f\left(u+\alpha_{n}\left(\delta_{n} u+\beta u+v\right)\right)-f(u)\right]=D f(u)(\beta u+v)+\varphi\left(\alpha_{n}, \delta_{n}\right),
$$

where $\varphi\left(\alpha_{n}, \delta_{n}\right)=\alpha_{n}^{-1}\left[f\left(u+\alpha_{n}\left(\delta_{n} u+\beta u+v\right)\right)-f(u)\right]-D f(u)(\beta u+v)$.

Since $\lim _{n \rightarrow \infty} \varphi\left(\alpha_{n}, \delta_{n}\right)=0$, letting $n$ tend to $\infty$ we obtain

$$
\begin{aligned}
0 \leq D f(u)(\beta u+v) & =-\frac{D g(u)(v)}{D g(u)(u)} D f(u)(u)+D f(u)(v) \\
& =D f(u)(v)-\lambda D g(u)(v)
\end{aligned}
$$


where $\lambda=\frac{D f(u)(u)}{D g(u)(u)}$. Thus for any $v$ in $C \cap G$,

$$
\lambda D g(u)(v-u)=\lambda D g(u)(v)-D f(u)(u) \leq D f(u)(v)-D f(u)(u) .
$$

Theorem 2.6. Let $U$ be an open subset of a topological vector space $E, G$ be a linear subspace of $E, x_{0}$ be in $U, r$ be a real number, and $f$ and $g$ be two functions from $U$ into $\mathbb{R}$. Put $M=\{x \in U: g(x)=r\}$. Assume that:

(i) $f\left(x_{0}\right)$ is the minimum (or maximum) of $f(M)$,

(ii) $f$ is strongly $G$-differentiable at $x_{0}$ and $g$ is $G$-differentiable at $x_{0}$,

(iii) $g$ is $G$-continuous on $U$ and $D g\left(x_{0}\right) \neq 0$.

Then there exists a real number $\lambda$ such that $D f\left(x_{0}\right)(h)=\lambda D g\left(x_{0}\right)(h) \forall h \in G$.

Proof. Choose $w$ in $G$ such that $D g\left(x_{0}\right)(w)>0$, and fix a vector $v$ in $G$. Applying Lemma 2.3, we can find two sequences of real numbers $\left\{\alpha_{n}\right\}$ and $\left\{\delta_{n}\right\}$ converging to 0 such that $\alpha_{n}$ is positive and $g\left(u_{n}\right)=r$ for any integer $n$, where $u_{n}=u+$ $\alpha_{n}\left(\delta_{n}+\beta\right) w+\alpha_{n} v$ and $\beta=-\frac{D g(u)(v)}{D g(u)(w)}$. Since $U$ is open, we can suppose that $u_{n}$ is in $M$ for any integer $n$. Arguing as in the proof of Theorem 2.5, we obtain

$$
0 \leq\left[D f\left(x_{0}\right)-\lambda D g\left(x_{0}\right)\right](v) \text {. }
$$

Replacing $v$ in the above inequality by $-v$ we get the theorem.

Remark 2.7. Without using the classical implicit function theorem (see [7], p.362) in the proof of Theorem 2.6, we do not need the completeness of $E$, which is crucial in the classical Lagrange multiplier theorems.

We obtain a result on the signs of Lagrange multipliers in the following theorem.

Theorem 2.8. Let $U$ be an open subset of a topological vector space $E, G$ be a linear subspace of $E, x_{0}$ be in $U, r$ be a real number, $f$ and $g$ be two functions from $U$ into $\mathbb{R}$ and $M=\{x \in U: g(x) \geq r\}$. Assume that:

(i) $f\left(x_{0}\right)$ is the minimum of $f(M)$,

(ii) $f$ is strongly $G$-differentiable at $x_{0}$ and $g$ is $G$-differentiable at $x_{0}$,

(iii) $g$ is $G$-continuous on $U$ and $D g\left(x_{0}\right) \neq 0$.

Then there exists a non-negative real number $\lambda$ such that $D f\left(x_{0}\right)(h)=\lambda D g\left(x_{0}\right)(h)$ $\forall h \in G$.

Proof. Put $\alpha=g\left(x_{0}\right), S=\{x \in U: g(x)=\alpha\}$ and $N=\{x \in U: g(x) \geq \alpha\}$. We have $\alpha \geq r$ and

$$
f\left(x_{0}\right)=\min f(S)=\min f(N) .
$$

According to Theorem 2.6 there exists a real number $\lambda$ such that

$$
D f\left(x_{0}\right)(h)=\lambda D g\left(x_{0}\right)(h) \quad \forall h \in G .
$$

Now we prove that $\lambda \geq 0$. Let $k$ be in $G$ such that $D g\left(x_{0}\right)(k)>0$. Since $g$ is $G$-differentiable at $x_{0}$, we have

$$
\lim _{t \rightarrow 0} \frac{g\left(x_{0}+t k\right)-g\left(x_{0}\right)}{t}=D g\left(x_{0}\right)(k)>0 .
$$

Thus, there exists a $\theta>0$ such that for any $t \in(0, \theta)$ we have

$$
g\left(x_{0}+t k\right)-g\left(x_{0}\right)>0
$$


or $\left(x_{0}+t k\right) \in N$. Consequently, since $f$ is $G$-differentiable at $x_{0}$ and $f\left(x_{0}\right)=$ $\min f(N)$, we have

$$
0 \leq \lim _{t \rightarrow 0^{+}} \frac{f\left(x_{0}+t k\right)-f\left(x_{0}\right)}{t}=\operatorname{Df}\left(x_{0}\right)(k) .
$$

Since $D g\left(x_{0}\right) k>0$, combining (2.1) and (2.2) we see that $\lambda \geq 0$.

Denote by $M^{\prime}$ the set $\{x \in U: g(x) \leq r\}$. We note that if $f\left(x_{0}\right)=\max f\left(M^{\prime}\right)$, we obtain the same result. We have a similar result for $\lambda \leq 0$, if $f\left(x_{0}\right)=\max f(M)$, or $f\left(x_{0}\right)=\min f\left(M^{\prime}\right)$.

Remark 2.9. The signs of Lagrange multipliers are important to solve some problems. In [8] Le and Schmitt applied the regularity of solutions of elliptic equations to get the positivity of Lagrange multipliers. Their method may not be available if the equations are strongly singular. Our main idea in the proof of the above theorem is as follows : choose a convenient value level of function $g$ when we apply the Lagrange multiplier theorem.

\section{A LaX-Milgram theOREM}

In this section we point out that the Lax-Milgram theorem can be obtained by using the Lagrange multipliers theorem. Furthermore, applying techniques in section 2 we get the following version of Lax-Milgram's theorem.

Theorem 3.1. Let $E$ be a reflexive Banach space, $\left\{E_{n}\right\}$ be an increasing sequence of closed subspaces of $E$, and $V$ be $\bigcup_{n=1}^{\infty} E_{n}$. Let $f$ be a function from $E \times V$ into $\mathbb{R}$ and $T$ be a bounded linear mapping from $V$ into $\mathbb{R}$, where the norm on $V$ is the one induced from $E$. Suppose the following conditions are satisfied.

(i) For fixed $n$ in $\mathbb{N},\left.f_{n} \equiv f\right|_{E_{n} \times E_{n}}$ is a bilinear bounded function, and for any fixed $v$ in $V, f(., v)$ is a linear bounded functional on $E$.

(ii) $f$ is coercive on $V$, i.e., there exists $C>0$ such that

$$
f(u, u) \geq C\|u\|^{2} \quad \forall u \in V .
$$

Then there exists a vector $z$ in $E$ such that

$$
f(z, v)=T(v) \quad \forall v \in V .
$$

Proof. Denote by $T_{n}$ the restriction of $T$ on $E_{n}$. If $T \equiv 0$, we choose $z=0$. If $T$ $\not \equiv 0$, we can suppose that $T_{1}^{-1}(\{1\})$ is not empty. Put $\phi_{n}(u)=\frac{1}{2} f_{n}(u, u)$ for any $u$ in $\in E_{n}$. It is clear that $\phi_{n}$ and $T_{n}$ are strongly $E_{n}$-differentiable. Now putting $S_{n}=T_{n}^{-1}(\{1\})$, we see that $S_{n} \subset S_{n+1}$ for all $n$ in $\mathbb{N}$. Since $T_{n}$ is a linear bounded functional on $E_{n}$, it is weakly continuous on $E_{n}$. By Theorem 1.2 in ([13, p.4), there exists $u_{n}$ in $E_{n}$ such that $\phi_{n}\left(u_{n}\right)=\min \phi_{n}\left(S_{n}\right) \forall n \in \mathbb{N}$. By Theorem [2.6] there exists a number $\lambda_{n}$ such that $D \phi_{n}\left(u_{n}\right)(v)=\lambda_{n} D T_{n}\left(u_{n}\right)(v) \forall v \in E_{n}$ or

$$
f\left(u_{n}, v\right)=\lambda_{n} T(v) \quad \forall v \in E_{n}, n \in \mathbb{N},
$$

It follows that $\lambda_{n}=f\left(u_{n}, v\right)$ for any $v$ in $S_{n}$ and

$$
0<C\left\|u_{n}\right\|^{2} \leq f_{n}\left(u_{n}, u_{n}\right)=\lambda_{n} .
$$

Since $T$ is a bounded linear mapping on $V$, there is a positive real number $\beta$ such that

$$
\beta<\|x\|^{2} \quad \forall x \in T^{-1}(1) .
$$


Combining (3.2) and (3.3) we get a positive real number $\alpha$ such that $\lambda_{n}>\alpha$ for any integer $n$. Put $z_{n}=\lambda_{n}^{-1} u_{n}$ for any integer $n$. Because $S_{n} \subset S_{n+1}$ and $\phi\left(u_{n}\right)$ $=\min \phi\left(S_{n}\right)$, we see that $\left\{\phi\left(u_{n}\right)\right\}_{n}$ is non-increasing. Therefore,

$$
C\left\|u_{n}\right\|^{2} \leq 2 \phi\left(u_{1}\right) \text {. }
$$

This implies that $\left\{\left\|u_{n}\right\|_{E}\right\}$ is bounded in $\mathbb{R}$. Since $E$ is a reflexive Banach space, we can assume that $\left\{u_{n}\right\}$ weakly converges to a vector $u$ in $E$. Fix a $w$ in $S_{1}$. Since $f(., w)$ belongs to the dual space of $E$, we have $\lim _{n \rightarrow \infty} f\left(u_{n}, w\right)=f(u, w)$. Thus $\lim _{n \rightarrow \infty} \lambda_{n}=\lambda \equiv f(u, w)$. Therefore, $\lambda \geq \alpha>0$ and $\left\{z_{n}\right\}$ weakly converges to $z$ $\equiv \lambda^{-1} u$ in $E$.

Now fix a $v$ in $V$. There is an integer number $n_{0}$ such that $v \in E_{n}$ for any $n \geq$ $n_{0}$. By $(3.1)$,

$$
f\left(z_{n}, v\right)=T(v) \quad \forall n \geq n_{0} .
$$

Letting $n$ tend to infinity, by $(i)$, we get the theorem.

Remark 3.2. The vector $z$ in the above theorem may not be in $V$ (see Theorem 3.3 in this paper). If $V=E$ and $f$ is well-defined and continuous on $E \times E$, Theorem 3.1 has been proved in [10, 11].

Let $\Omega$ be a bounded domain in $\mathbb{R}^{m}$, and let $A=\left(A_{i j}^{\alpha \beta}\right)$ be a measurable matrix function from $\Omega$ into $\mathbb{R}^{N^{2} \times m^{2}}$ such that $A_{i j}^{\alpha \beta}=A_{i j}^{\beta \alpha}$ for any $i, j=1, \cdots, N$, and any $\alpha, \beta=1, \cdots, m$. We assume that

(i) There is a function $\rho$ in $L_{l o c}^{\infty}(\Omega)$ such that

$$
\|A(x)\| \leq \rho(x) \quad \forall x \in \Omega .
$$

(ii) There is a positive real number $\nu$ such that

$$
A_{i j}^{\alpha \beta}(x) \xi_{\alpha}^{i} \xi_{\beta}^{j} \geq \nu|\xi|^{2} \quad \forall(x, \xi) \in \Omega \times \mathbb{R}^{N \times m} .
$$

By convention, repeated Greek indices are summed from 1 to $m$, Latin indices from 1 to $N$.

Applying Theorem 3.1 we get the following result.

Theorem 3.3. Let $h$ be in $L^{s}\left(\Omega, \mathbb{R}^{N}\right)$, where $s>1$ if $m=2$ and $s=\frac{2 m}{m+2}$ if $m$ $\geq 3$. Then there exists a generalized solution $u_{0}$ in $W_{0}^{1,2}\left(\Omega, \mathbb{R}^{N}\right)$ to the following boundary problem:

$$
\left\{\begin{array}{l}
-\partial_{\beta}\left(A_{i j}^{\alpha \beta} \partial_{\alpha} u^{i}\right)=h^{j} \quad \text { on } \Omega, \forall j=1, \cdots, N, \\
\left.u\right|_{\partial \Omega}=0 .
\end{array}\right.
$$

Proof. Let $\left\{\Omega_{n}\right\}$ be an increasing sequence of open subsets in $\Omega$ such that $\bar{\Omega}_{n}$ is contained in $\Omega_{n+1}$ and $\Omega=\bigcup_{n=1}^{\infty} \Omega_{n}$. Let $E$ be the usual Sobolev space $W_{0}^{1,2}\left(\Omega, \mathbb{R}^{N}\right)$, $E_{n}=W_{0}^{1,2}\left(\Omega_{n}, \mathbb{R}^{N}\right)$ for any integer $n$ and

$$
\begin{aligned}
V & =\bigcup_{n=1}^{\infty} E_{n}, \\
f(u, v) & =\int_{\Omega} A_{i j}^{\alpha \beta} \partial_{\alpha} u^{i} \partial_{\beta} v^{j} d x \quad \forall(u, v) \in E \times V .
\end{aligned}
$$


By the Sobolev embedding theorem we can define

$$
T(v)=\int_{\Omega} h^{j} v^{j} d x \quad \forall v \in E,
$$

and see that $T$ is a bounded linear mapping from $E$ into $\mathbb{R}$. Applying the foregoing theorem we can find a function $u_{0}$ in $E$ such that

$$
f\left(u_{0}, v\right)=T(v) \quad \forall v \in V .
$$

We get the result by using $v=\left(\delta_{j}^{1}, \cdots, \delta_{j}^{N}\right) \varphi$ in (3.4), where $\varphi$ is in $C_{c}^{\infty}(\Omega), j$ is in $\{1, \cdots, N\}$, and $\delta_{j}^{k}$ is the Kronecker number.

\section{An APPLICATION to VARIATIONAL INEQUALITIES}

Denote by $X$ the space of all $u$ in the Sobolev space $W^{2,2}((0, l))$ such that $u$ and $u^{\prime}$ are in $W_{0}^{1,2}((0, l))$. Then $X$ is a normed space with the $W^{2,2}((0, l))$-norm. Fixing an integrable function $P$ on $(0, l)$, for any $u$ in $X$ we define

$$
f(u)=\frac{1}{2} \int_{0}^{l}\left|u^{\prime \prime}(x)\right|^{2} d x \quad \text { and } \quad g(u)=\frac{1}{2} \int_{0}^{l} P(x)\left|u^{\prime}(x)\right|^{2} d x .
$$

Let $C$ be a closed and convex cone of $X$ such that there exists $u_{0}$ in $C$ with $g\left(u_{0}\right)=r \neq 0$. We are seeking solutions $(u, \lambda)$ in $C \times \mathbb{R} \backslash\{0\}$ such that

$$
D f(u)(v-u) \geq \lambda D g(u)(v-u) \quad \forall v \in C .
$$

Put $M=\{u \in C: g(u)=r\}=C \cap g^{-1}(\{r\})$.

Lemma 4.1. Under the above conditions we have:

(i) $f$ is weakly lower semi-continuous, coercive and strongly $X$-differentiable on $X$ and

$$
D f(u)(h)=\int_{0}^{l} u^{\prime \prime} h^{\prime \prime} d x \quad \forall u, h \in X .
$$

(ii) $g$ is weakly continuous and $X$-differentiable on $X$ and

$$
D g(u)(h)=\int_{0}^{l} P u^{\prime} h^{\prime} d x \quad \forall u, h \in X .
$$

Proof. By the Poincaré inequality $|f(u)|^{1 / 2}$ is equivalent to the norm on $X$. Therefore $f$ is weakly lower semi-continuous and coercive on $X$. For any $u, h, k$ in $X$ and any $s, t$ in $\mathbb{R}$ we have

$$
\begin{aligned}
& f(u+s t h+s k)-f(u)=\int_{0}^{l} s u^{\prime \prime}\left(t h^{\prime \prime}+k^{\prime \prime}\right) d x+\frac{1}{2} \int_{0}^{l} s^{2}\left|\left(t h^{\prime \prime}+k^{\prime \prime}\right)\right|^{2} d x, \\
& \lim _{(s, t) \rightarrow 0} \frac{f(u+s t h+s k)-f(u)}{s}=\int_{0}^{l} u^{\prime \prime} k^{\prime \prime} d x .
\end{aligned}
$$

Let $\left\{u_{n}\right\}$ be an arbitrary sequence converging weakly to $u$ in $X$. Then $\left\{\left\|u_{n}\right\|_{X}\right\}$ is bounded. Because of the compactness of the embedding from $W^{2,2}((0, l))$ into $C^{1}([0, l])$, there exists a subsequence $\left\{u_{n_{k}}\right\}$ of $\left\{u_{n}\right\}$ converging to $u$ in $C^{1}([0, l])$, 
and thus $\left\{u_{n_{k}}^{\prime}\right\}$ converges to $u^{\prime}$ in $C([0, l])$. Moreover $\left\{u_{n_{k}}^{\prime}\right\}$ is bounded in $C([0, l])$. Since $P \in L^{1}((0, l))$, we have

$$
\begin{aligned}
& \left|g\left(u_{n_{k}}\right)-g(u)\right|=\frac{1}{2}\left|\int_{0}^{l} P\left(\left|u_{n_{k}}^{\prime}\right|^{2}-\left|u^{\prime}\right|^{2}\right) d x\right| \leq \frac{1}{2} \int_{0}^{l}|P|\left(\left|u_{n_{k}}^{\prime}\right|+\left|u^{\prime}\right|\right)\left(\left|u_{n_{k}}^{\prime}-u^{\prime}\right|\right) d x \\
& \quad \leq C \int_{0}^{l}|P| \sup _{x} \in[0, l] \\
& \quad\left|\left(u_{n_{k}}^{\prime}-u^{\prime}\right)(x)\right| d x \leq C\|P\|_{L^{1}((0, l))}\left\|\left(u_{n_{k}}^{\prime}-u^{\prime}\right)\right\|_{C([0, l])} .
\end{aligned}
$$

Therefore for any sequence $\left\{u_{n}\right\}$ weakly converging to $u$ we can find a subsequence $\left\{u_{n_{k}}\right\}$ such that $\left\{g\left(u_{n_{k}}\right)\right\}$ converges to $g(u)$. This implies that $\left\{g\left(u_{n}\right)\right\}$ converges to $g(u)$ and $g$ is weakly continuous.

For any $u, h$ in $X$ and any $t$ in $\mathbb{R}$ we have

$$
g(u+t h)-g(u)=t \int_{0}^{l} P u^{\prime} h^{\prime} d x+\frac{1}{2} t^{2} \int_{0}^{l} P\left|h^{\prime}\right|^{2} d x .
$$

Thus

$$
\lim _{t \rightarrow 0} \frac{g(u+t h)-g(u)}{t}=\int_{0}^{l} P u^{\prime} h^{\prime} d x
$$

Theorem 4.2. Under the above conditions there exists a solution $(u, \lambda)$ of $(Q)$.

Proof. Since $g$ is weakly continuous, $g^{-1}(\{r\})$ is a weakly closed subset of $X$. From Theorem III.7 in [4], since $C$ is closed and convex, we have that $C$ is weakly closed. Consequently, $M=C \cap g^{-1}(\{r\})$ is weakly closed. Therefore, by Lemma 4.1 (i) and Theorem 1.2 in [13] there exists a function $u \in M$ such that

$$
f(u)=\min _{v \in M} f(v) \text {. }
$$

Moreover, since $g(u)=r \neq 0$, we have $D g(u)(u)=2 g(u) \neq 0$. By Lemma 4.1 and Theorem 2.5 we obtain the variational inequality (Q) with $\lambda=\frac{D f(u)(u)}{D g(u)(u)}$, which is not equal to 0 .

Remark 4.3. If $P>0$, the real number $\lambda$ is also positive. If $P \equiv 1$ the problem (Q) is solved in Section 64.6 in [14].

\section{A quasilinear elliptic eigenvalue Problem}

Denote by $E$ and $G$ the spaces $W_{0}^{1,2}\left(\Omega, \mathbb{R}^{N}\right)$ and $C_{0}^{\infty}\left(\Omega, \mathbb{R}^{N}\right)$ respectively, where $\Omega$ is a bounded domain in $\mathbb{R}^{m}$. Let $g$ be a real functional on $E$, and let $\left(a^{\alpha \beta}\right)_{1 \leq \alpha, \beta \leq m}$ be a symmetric, and bounded matrix function from $\Omega \times \mathbb{R}^{N}$ into $\mathbb{R}^{m \times m}$. We assume that

(A1) $a^{\alpha \beta}$ is measurable in $x \in \Omega$, differentiable in $u \in \mathbb{R}^{N}$, and for a.e $x$, $\partial_{u} a^{\alpha \beta}(x,$.$) is uniformly continuous in u$, uniformly in $x$. Moreover, there exist a function $\rho$ in $L_{l o c}^{2}(\Omega)$ and a constant $c$ such that $|f(x, u)| \leq c$ and $\left|\partial_{u} f(x, u)\right| \leq \rho(x)$ for any $(x, u)$ in $\Omega \times \mathbb{R}^{N}$.

$(A 2)$ There is a positive real number $\nu$ such that $a^{\alpha \beta}(x, u) \xi_{\alpha}^{i} \xi_{\beta}^{i} \geq \nu|\xi|^{2} \forall(x, u, \xi)$ $\in \Omega \times \mathbb{R}^{N} \times \mathbb{R}^{N m}$.

$(A 3) s^{2} a^{\alpha \beta}(x, s u) \xi_{\alpha}^{i} \xi_{\beta}^{i} \leq a^{\alpha \beta}(x, u) \xi_{\alpha}^{i} \xi_{\beta}^{i} \forall(x, u, \xi, s) \in \Omega \times \mathbb{R}^{N} \times \mathbb{R}^{N m} \times[0,1]$. 
$(G 1) g$ is continuous with respect to weak convergence topology in $E, g(0)=0$ and $M=\{x \in E: g(x) \geq 1\}$ is not empty.

(G2) $g$ is $G$-differentiable on $E$, and $\nabla g(u) \neq 0$ if $g(u) \neq 0$.

Put $f(u)=\frac{1}{2} \int_{\Omega} a^{\alpha \beta}(x, u(x)) \partial_{\alpha} u^{i}(x) \partial_{\beta} u^{i}(x) d x \forall u \in E$.

We have the following result.

Theorem 5.1. Under the conditions $(A 1),(A 2),(A 3),(G 1)$ and $(G 2)$ there exist a nonnegative real number $\lambda$ and $u$ in $E$ such that $g(u)=1$ and $\nabla f(u)=\lambda \nabla g(u)$.

Proof. Arguing as in the proof of Lemma 3.1 in 12 we see that $f$ is strongly $G$-differentiable on $E$. Let $\left\{u_{n}\right\}$ be a sequence in $M$ such that $\lim _{n \rightarrow \infty} f\left(x_{n}\right)=$ inf $f(M)$. Using $(A 2)$ and the Sobolev embedding theorem we can suppose that $\left\{u_{n}\right\}$ converges weakly to $v_{0}$ in $E$ and $\left\{u_{n}\right\}$ converges to $v_{0}$ a.e on $\Omega$. By $(A 1)$, Fatou's lemma and $(G 1)$ we see that $v_{0}$ belongs to $M$ and $f\left(v_{0}\right)=\min f(M)$. By $(G 1)$ and $(A 3)$ there is $s$ in $[0,1]$ such that $g\left(s v_{0}\right)=1$. Put $u_{0}=s v_{0}$. We see that $u_{0} \in M$ and $f\left(u_{0}\right)=\min f(M)$. Using $(G 2)$ we can apply Theorem 2.8 to get the theorem.

Remark 5.2. In 12 the following condition was considered:

(A4) There exists a positive real number $\nu^{\star} \leq \nu$ such that

$$
-u . \partial_{u} a^{\alpha \beta}(x, u) \xi_{\alpha}^{i} \xi_{\beta}^{i} \leq 2 \nu^{\star}|\xi|^{2} \quad \forall(x, u, \xi) \in \Omega \times \mathbb{R}^{N} \times \mathbb{R}^{N m} .
$$

Now assume that $\left(a^{\alpha \beta}\right)_{1 \leq \alpha, \beta \leq m}$ satisfies $(A 4)$. We prove that it satisfies $(A 3)$. Indeed, letting $x$ be in $\Omega \times \mathbb{R}^{N} \times \mathbb{R}^{N m} \times[0,1]$, we have

$$
\begin{gathered}
s^{2} a^{\alpha \beta}(x, s u) \xi_{\alpha}^{i} \xi_{\beta}^{i}=s^{2} a^{\alpha \beta}(x, u) \xi_{\alpha}^{i} \xi_{\beta}^{i} \\
+s^{2} \int_{0}^{1} \partial_{u} a^{\alpha \beta}(x, u+t(s-1) u)(s-1) u \xi_{\alpha}^{i} \xi_{\beta}^{i} d t \\
=a^{\alpha \beta}(x, u) \xi_{\alpha}^{i} \xi_{\beta}^{i}+\left(s^{2}-1\right) a^{\alpha \beta}(x, u) \xi_{\alpha}^{i} \xi_{\beta}^{i} \\
+s^{2} \int_{0}^{1} \frac{-(1-s)}{1+t(s-1)} \partial_{u} a^{\alpha \beta}(x,[1+t(s-1)] u)[1+t(s-1)] u \xi_{\alpha}^{i} \xi_{\beta}^{i} d t \\
\leq a^{\alpha \beta}(x, u) \xi_{\alpha}^{i} \xi_{\beta}^{i}+\left(s^{2}-1\right) \nu|\xi|^{2}+2 s^{2} \frac{1-s}{s} \nu^{\star}|\xi|^{2} \\
\leq a^{\alpha \beta}(x, u) \xi_{\alpha}^{i} \xi_{\beta}^{i}-(s-1)^{2} \nu|\xi|^{2} \leq a^{\alpha \beta}(x, u) \xi_{\alpha}^{i} \xi_{\beta}^{i} .
\end{gathered}
$$

Therefore we can get Theorem 3 in [12] with relaxation of compactness and some other conditions.

Our results may be applied to $p$-Laplace equations (see [5]).

\section{ACKNOWLEDGMENTS}

The authors would like to thank the referees for very helpful comments. The third author would like to thank Professors Jose Seade and Alberto Verjovsky for the kind hospitality at the Instituto de Matematicas U.N.A.M., Cuernavaca, Morelos, Mexico. 


\section{REFERENCES}

[1] R.A. Adams. Sobolev Spaces Academic Press, New York, 1975. MR0450957 (56:9247)

[2] D. Arcoya and L. Boccardo. Critical points for multiple integrals of calculus of variations Arch. Rat. Mech. Anal., 134(1996), 249-274. MR.1412429 (97h:49003)

[3] D. Arcoya and L. Boccardo. Some remarks on critical point theory for nondifferentiable functionals Nonlinear Differ. Equ. Appl., 6(1999), 79-100. MR.1674782 (2000c:58018)

[4] H. Brezis. Analyse Fonctionelle (Masson,Paris) 1987.

[5] P.X. Du and D.M.Duc. A non-homogenuous p-Laplace equation in border case. Acta Mathematica Vietnamica, 27(2002) 69-75. MR,1914409 (2003b:35082)

[6] D.M.Duc. Nonlinear Singular Elliptic Equations J.London.Math.Soc. 40 (1989) 420-440. MR1053612 (91g:35107)

[7] S. Lang. Analysis I. Addison-Wesley, Reading, 1969.

[8] V.K.Le and K.Schmitt. Minimization problems for noncoercive functionals subject to constraints Transactions Amer. Math. Soc., 347(1995), 4485-4513. MR1316854 (96d:49007)

[9] B. Pellacci. Critical points for some functionals of the calulus of variations. Topol. Methods Nonlinear Anal. 17(2001), 285-305.. MR.1868902 (2002j:49025)

[10] S. Ramaswamy. The Lax-Milgram theorem for Banach spaces. I Proc. Japan Acad. 56 (1980) 462-464. MR0605763 (82e:47015a)

[11] S. Ramaswamy. The Lax-Milgram theorem for Banach spaces. II Proc. Japan Acad. 57 (1981) 29-33. MR0608386 (82e:47015b)

[12] M.Struwe. Quasilinear elliptic eigenvalue problems Comment. Math. Helvetici. 58 (1983) 509-527. MR0727715 (85h:58036)

[13] M.Struwe. Variational Methods, Springer-Verlag, New York, 1996.

[14] E.Zeidler. Nonlinear Functional Analysis and its Applications, Vol.3, Springer-Verlag, New York, 1983.

Department of Mathematics, University of Utah, Salt lake City, Utah 84112

E-mail address: anle@math.utah.edu

Department of Mathematics, Indiana University, Bloomington, Indiana 47405

E-mail address: dxpham@indiana.edu

Department of Mathematics, Informatics, National University of Hochiminh City, VIETNAM

E-mail address: dmduc@hcmc.netnam.vn

School of Mathematics, University of Minnesota, Minneapolis, Minnesota 55455

E-mail address: phan@math.umn.edu 